stimulus of dissatisfaction, leading to improved techniques and machines (and, incidentally, providing an excellent yardstick of present performance against which alleged improvements can be compared). Mechanization has a tendency to induce complacency and 'freeze' techniques, and active quality control programmes are an important counter-measure.

For repeated tests of reproducibility, one or more pools of test material are required. This should be as similar as possible to the material normally analysed; if, for example, blood plasma is the material normally analysed, simple aqueous solutions are not satisfactory as control materials, although they may be adequate as calibration standards (Whitby, Mitchell $\&$ Moss, 1967 ). The pool of test material must be stable for long periods, and if this is unattainable some compromise may be required, e.g. use of aqueous control solutions as well as standards, coupled with re-analysis, in each batch, of a number of samples which were analysed in the previous batch. It is absolutely essential that the control material be homogeneous, otherwise one is testing for overall reproducibility of both the sampling and the analysing steps, and (in biological materials especially) these are really separate problems which should be kept under separate but simultaneous review.

The day-to-day operation of the quality control system should be the responsibility of one person, of sufficient seniority and self-confidence to stop the operation of the laboratory when the control data warrant it, and able to direct the tracing and correction of whatever fault caused the control data to fall outside the permissible limits. In the mechanized laboratory, the machine operator should not also be the quality controller, but the latter must be continuously available and must deal with the results of the control analyses promptly. Control systems which generate data which are not promptly appraised, and acted on immediately when necessary, are both expensive and futile. Computer assistance should both simplify and speed up the whole process of control of analytical quality, but it is far from being a sine qua non, even in the extensively mechanized laboratory.

REFERENCE

Whitby, L. G., Mitchell, F. L. \& Moss, D. W. (1967). Adv. clin. Chem. 1o, 65.

\title{
Recent advances in amino acid analysis
}

By Alan J. Thomas, Department of Biochemistry, Imperial College, London, $S W 7$

The great interest in the nutritional and metabolic interrelationships of the amino acids has been stimulated by the increasing ease of separating and estimating them. Yet most available methods are still inadequate to provide the extent of information needed to sufficiently interpret the processes of digestion, absorption and conservation of protein in the animal. This is particularly true when one realizes that the really meaningful criterion in metabolic studies is turnover rather than static con- 
centration, and that therefore isotopic labelling and measurement must comprise an essential part of the technique.

An amino acid analysis system has been described (Thomas, 1970), completely automated from sample loading to data calculation, and with facilities to permit determination of ${ }^{14}$ carbon and ${ }^{15}$ nitrogen. This system was capable of seventy-four analyses/week, and was run by one skilled person who also prepared the samples.

Further improvements have resulted from reducing column length from $75 \mathrm{~cm}$ to $35 \mathrm{~cm}$, resin bead size from $\mathrm{I} 3 \mu \mathrm{m}$ to I $\mu \mathrm{m}$ and reducing the scan time of the gradient and control programmer from $4 \cdot 5 \mathrm{~h}$ to $\mathrm{I} \cdot 75 \mathrm{~h}$. The two chromatography columns have been redesigned to improve the effluent flow pattern. New colorimeters designed to accommodate flow cells with effective wash-out volumes reduced to $20 \mu \mathrm{l}$ have been introduced. The resulting increase in speed ( $\mathrm{r} \cdot 75 \mathrm{~h} / \mathrm{chromato-}$ gram) has raised the capacity to 192 samples/week, with no loss in resolution or increase in reagent consumption or operator time. Sharper peaks have increased sensitivity to the range 5-100 $\mathrm{n}$-moles/amino acid. The analytical data are presented on logarithmic recorders with servopotentiometers giving voltages directly proportional to absorbance. These voltages are manipulated with analogue techniques to give base-line compensation and correction, and fed to Honeywell Precision Integrators which print out peak areas on paper drawn out at the same speed as the recorder chart speed. Allocation of the printed areas to the appropriate amino acids is thus easily accomplished, and requires about $30 \mathrm{~min}$ for a day's output of twentyeight chromatograms. Calculation was formerly completed using an Olivetti Programmer IOI which, with colour values and arithmetic instructions stored on magnetic cards, accepted peak areas and produced: total yield; yield of each amino acid in $\mathrm{mg}$ and in $\mu$ moles and percentage composition of amino acids in the mixture. Calculation took over $5 \mathrm{~min} /$ chromatogram so that for $\mathrm{I} 92$ chromatograms this represented $\mathrm{i} 6 \mathrm{~h}$ of operator time each week. Currently, the peak areas and sample size are entered on IBM cards (about I min/chromatogram) and processed on an IBM 7094. The computer output expresses amino acids as: $\mathrm{mg} / \mathrm{I} / 0 \mathrm{~g}$ protein, $\mathrm{mg} / \mathrm{roo} \mathrm{g}$ dry weight and as molar percentage, and also gives: protein as percentage of sample, $\mathrm{mg} / \mathrm{I} 00 \mathrm{~g}$ ammonia- $\mathrm{N}$, and $\mathrm{mg} / \mathrm{I} 00 \mathrm{~g}$ total $\mathrm{N}$. The card stacks are retained and permit subsequent retrieval of information in any chosen form.

\section{REFERENCE}

Thomas, A. J. (1970). In Automation, Mechanisation and Data Handling in Microbiology p. 107. London: Academic Press. 